\title{
Amniotic fluid embolism: A Case of Successful Cardiac Ultrasound and Rotational Thromboelastometry (ROTEM) Guided Resuscitation in a Patient Undergoing Caesarean Section
}

\author{
S. Vrecic Slabe, Vitas Radisavljevic, K. Marusic Gaser, M. Lucovnik, G. Brzan \\ Department of Anaesthesiology and Surgical Intensive Therapy \\ The Division of Gynaecology and Obstetrics \\ University Medical Centre Ljubljana, Slovenia
}

\section{Background:}

Amniotic fluid embolism (AFE) is rare but life-threatening condition. Predominant characteristics are abrupt onset of respiratory failure, circulatory collapse and disseminated intravascular coagulopathy (DIC). The symptoms occur in the peripartum period due to the inflow of amniotic components into the maternal circulation $(1,2)$.

We present a patient with an AFE who developed hypoxia with bronchospasm, hypotension and coagulopathy during CS procedure. Intraoperative use of cardiac ultrasound and ROTEM guided resuscitation resulted in fully patient recovery.

\section{Case report:}

A 27-year old primigravida at $315 / 7$ weeks of gestation underwent urgent CS due to suspected placental abruption (vaginal bleeding, tachysystole and signs of fetal distress on fetal heath rate monitor)

After induction of anaesthesia and intubation, the drop in oxygen blood saturation and increased airway pressure was observed.

The patient was treated for suspected bronchospasm with salbutamol and aminophylline. Hypotension was detected, coupled with rapid blood pressure drop and loss of systolic measurement. Obstetrician performed the urgent neonate extraction. A male neonate weighting 2060 grams was born (Apgar score 5 at birth and 7 at 5 minutes of life, $\mathrm{pH}$ in the umbilical artery 7.07).

Because of potential anaphylactic reaction, tachycardic and hypotensive patient was treated with the infusion of adrenaline. Cardiac ultrasound showed normal right ventricle size with normal pressure, small, completely empty and hyperdymanic left ventricle, vena cava inferior was collapsed.

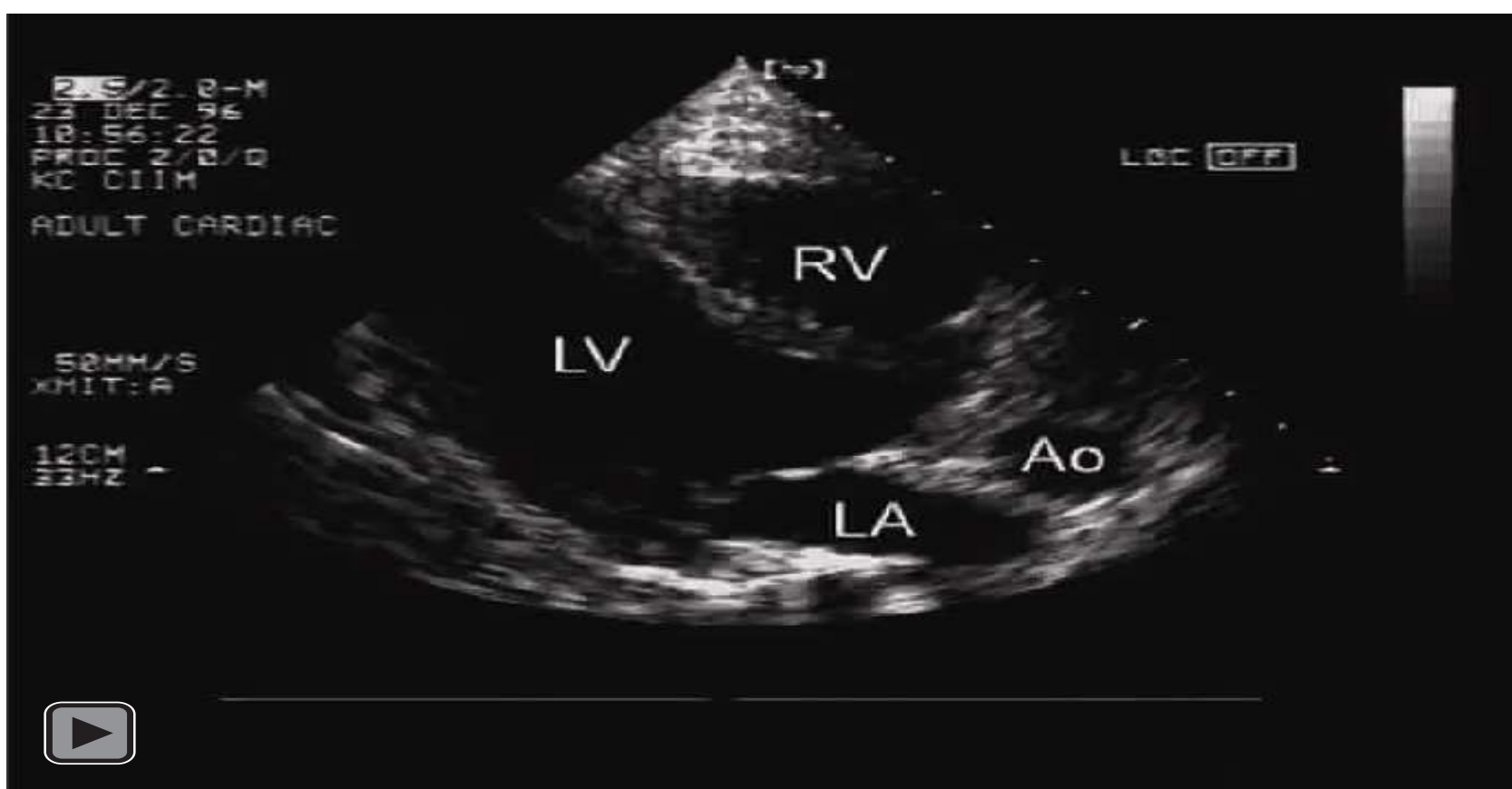

Figure1. ECHO in hyperdynamic circulation

Fluid resuscitation and infusion of phenylephrine was started, followed by norepinephrine infusion.

Blood for laboratory examination was sampled and blood for transfusion ordered.

After exclusion of pulmonary embolism, the probability of AFE resulted very high. The CS procedure completed with insignificant blood loss.

Within 90 minutes patient was cardiocirculatory stable.

\section{References:}

Tamura N, Farhana M, Oda T, Itoh $\mathrm{H}$, Kanayama N. Amniotic fluid embolism: Pathophysiology from the perspective of pathology J Obstet G Qdat,

Hurwich M, Zimmer D, Guerra E, Evans E, Shire T, Abernathy M, Shreve JT, Kolettis GR, McCurdy MT, Castellino FJ, Walsh M. A Case of Successful Thromboelastographic Guided Resuscitation after Postpartum Hemorrhage and Cardiac Arrest. J Extra Corpor Technol. 2016 Dec;48(4):194-197.
When CS procedure was completed, bleeding started from the wound. General oedema appeared. At the same time, ROTEM analysis indicated fibrinolysis and lab blood examination results showed hemoconcentration.

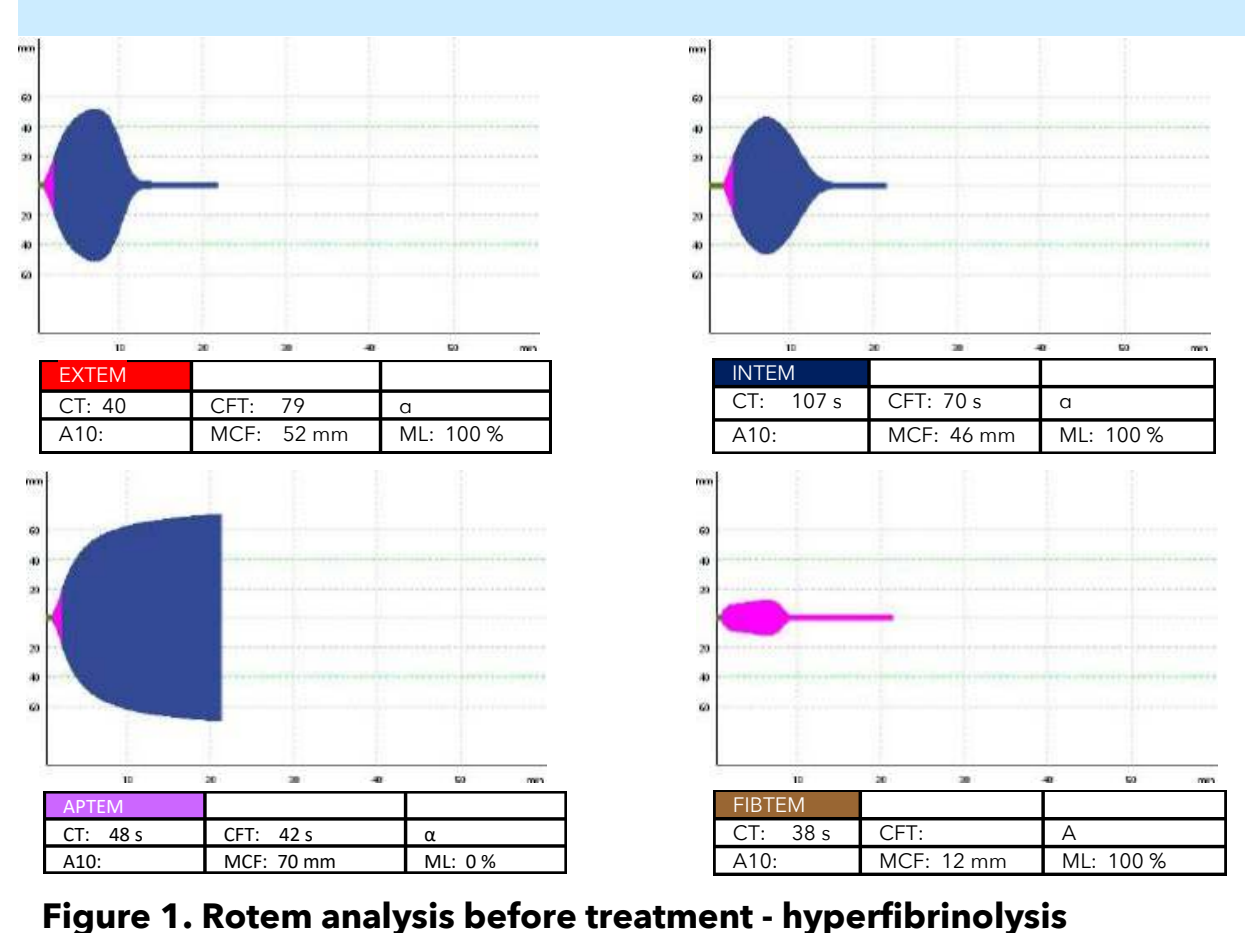

Tranexamic acid was applied to stop bleeding. Sedated patient was transported to the ICU.

After 12 hours, she was extubated and transferred back to maternity ward.
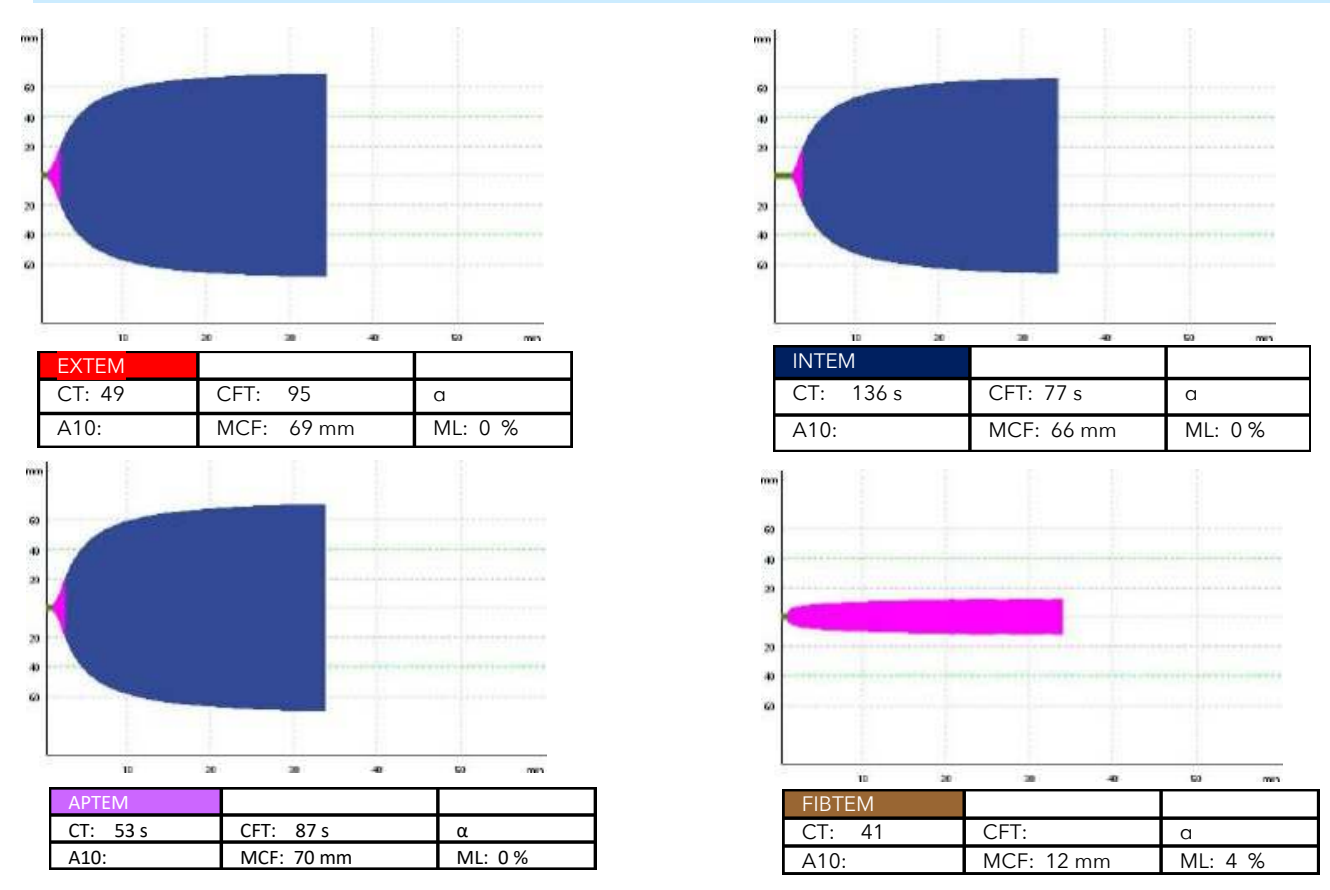

Figure 2. Rotem analysis after administration of tranexamic acid

\section{Discussion:}

AFE represents challenging situation for the anaesthesiologist with unpredictable course such as respiratory and circulatory failure and DIC.

The underlying reason for cardiocirculatory collapse in our case remain uncertain. Cardiac ultrasound might become one of the powerful tools to answer clinical questions and guide subsequent management in obsteric patient (3).

For point of care therapy in peripartum haemorrhage and coagulopathy ROTEM is essential. The administration of blood products was guided by ROTEM.

\section{Learning points:}

An ideal anaesthetic management of AFE consensus is yet to be reached.

Cardiac ultrasound examination and ROTEM results guide to better decision making and faster patient treatment. Both techniques contributed to successfully solved case 